\title{
Average Speed in Projectile Motion and in General Motion of a Particle
}

\author{
Chloe T. Calderon, Pirooz Mohazzabi \\ Department of Mathematics and Physics, University of Wisconsin-Parkside, Kenosha, WI, USA \\ Email:mohazzab@uwp.edu
}

How to cite this paper: Calderon, C.T. and Mohazzabi, P. (2018) Average Speed in Projectile Motion and in General Motion of a Particle. Journal of Applied Mathematics and Physics, 6, 1540-1548.

https://doi.org/10.4236/jamp.2018.67130

Received: July 3, 2018

Accepted: July 24, 2018

Published: July 27, 2018

Copyright $\odot 2018$ by authors and Scientific Research Publishing Inc. This work is licensed under the Creative Commons Attribution International License (CC BY 4.0).

http://creativecommons.org/licenses/by/4.0/

(c) (i) Open Access

\begin{abstract}
We calculate the average speed of a projectile in the absence of air resistance, a quantity that is missing from the treatment of the problem in the literature. We then show that this quantity is equal to the time-average instantaneous speed of the projectile, but different from its space-average instantaneous speed. It is then shown that this behavior is shared by general motion of all particles regardless of the dimensionality of motion and the nature of the forces involved. The equality of average speed and time-average instantaneous speed can be useful in situations where the calculation of one is more difficult than the other. Thus, making it more efficient to calculate one by calculating the other.
\end{abstract}

\section{Keywords}

Average Speed, Time-Average, Space-Average, Instantaneous Speed, Projectile Motion, General Motion

\section{Introduction}

Projectile motion, one of the simplest examples of two-dimensional motion in classical mechanics, has been extensively studied and thoroughly discussed in the literature and textbooks at different levels. Introductory treatment of projectile motion normally ignores air resistance and treats the problem using either differential and integral calculus [1]-[6] or algebra and trigonometry [7] [8], or even without trigonometry [9]. More advanced treatments of the problem include air resistance [10] [11] [12] [13]. However, to the best of the author's knowledge and based on extensive literature search, the average speed of a projectile during its motion has not been discussed in the literature at any level. Although this may seem trivial at first sight, it is an important concept in correlating different methods of averaging the speed of an object during its 
motion as will be explained below.

The average speed of a moving particle is defined as the total distance traveled $L$ divided by the total time $T$,

$$
S_{\text {ave }}=\frac{L}{T}
$$

and the instantaneous speed is defined as magnitude of the instantaneous velocity of the particle at a given time,

$$
v=|\mathbf{v}(t)|=\left|\frac{\mathrm{d} \mathbf{r}(t)}{\mathrm{d} t}\right|
$$

where $\mathbf{r}(t)$ is the position vector of the particle.

A question that occasionally comes up in discussions related to speed is whether the average of the instantaneous speed of an object during its motion is the same as its average speed. In general, the answer is definitely NO. For example, if an object travels half of a distance with speed $v_{1}$ and the other half with speed $v_{2}$, the average of these speeds is $\left(v_{1}+v_{2}\right) / 2$ whereas the average speed which, by definition is the total distance divided by total time, can be shown to be given by

$$
s_{\text {ave }}=\frac{2 v_{1} v_{2}}{v_{1}+v_{2}}
$$

Later on in this article we shall refer to the former average as the space-average of the speed.

In what follows, we show that the space-average of instantaneous speed of a particle is not, in general, equal to its average speed, however, the time-average of instantaneous speed is always equal to the average speed regardless of the nature of the motion. But first, we find the average speed, the time-average of instantaneous speed, and the space-average of instantaneous speed for a projectile in the absence of air resistance.

\section{Projectile Motion}

\subsection{Average Speed}

In the absence of air resistance, the components of the equation of motion for a projectile launched with an initial speed $v_{0}$ at an angle of elevation $\theta_{0}$ (Figure 1) are given by

$$
\begin{aligned}
& x=v_{0 x} t, \quad y=-\frac{1}{2} g t^{2}+v_{0 y} t \\
& v_{x}=v_{0 x}, \quad v_{y}=-g t+v_{0 y}
\end{aligned}
$$

where $v_{0 x}=v_{0} \cos \theta_{0}$ and $v_{0 y}=v_{0} \sin \theta_{0}$. Elimination of $t$ between the equations for $x$ and $y$ results in the equation of the trajectory,

$$
y=-\frac{g}{2 v_{0 x}^{2}} x^{2}+\frac{v_{0 y}}{v_{0 x}} x
$$

Defining the constants of the motion, 


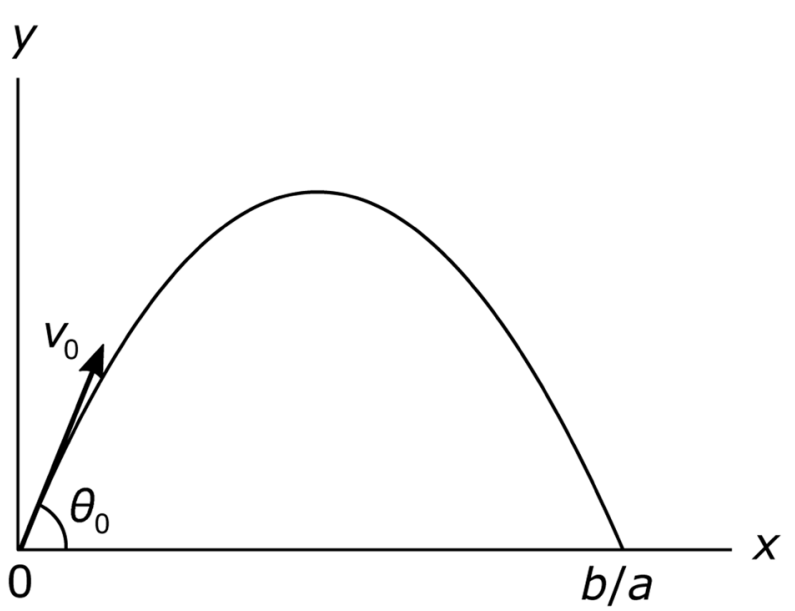

Figure 1. Trajectory of a projectile in the absence of air resistance. The constants $a$ and $b$ are defined by Equation (6).

$$
a=\frac{g}{2 v_{0 x}^{2}} \text { and } b=\frac{v_{0 y}}{v_{0 x}}
$$

the equation of the trajectory reduces to

$$
y=-a x^{2}+b x
$$

which is the equation of a parabola with $x$ intercepts at $x=0$ and $x=b / a$, as shown in Figure 1.

The element of arc length of a plane curve with equation $y=f(x)$ is given by

$$
\mathrm{d} L=\sqrt{1+\left(\frac{\mathrm{d} y}{\mathrm{~d} x}\right)^{2}} \mathrm{~d} x
$$

Using Equation (7), we obtain

$$
\mathrm{d} L=\sqrt{1+(2 a x-b)^{2}} \mathrm{~d} x
$$

Therefore, the total distance traveled by the projectile during its flight (returning to the same level from which it was launched) is given by

$$
L=\int_{0}^{b / a} \sqrt{1+(2 a x-b)^{2}} \mathrm{~d} x
$$

Finally, changing the variable to $u=2 a x-b$, we obtain

$$
L=\frac{1}{2 a} \int_{-b}^{b} \sqrt{1+u^{2}} \mathrm{~d} u=\frac{1}{a} \int_{0}^{b} \sqrt{1+u^{2}} \mathrm{~d} u
$$

Evaluation of this integral is elementary and can be found in any table of integrals [14]. Thus, we obtain

$$
L=\frac{1}{2 a}\left[b \sqrt{1+b^{2}}+\ln \left(b+\sqrt{1+b^{2}}\right)\right]
$$

Note that $b$ is a dimensionless quantity, and $a$ has the dimension of reciprocal length. 
From the second of Equation (4), the total time of flight can be calculated by setting $y=0$,

$$
T=\frac{2 v_{0 y}}{g}
$$

Therefore, the average speed of the projectile during its entire flight is

$$
s_{\text {ave }}=\frac{L}{T}
$$

Using Equation (12) and Equation (13) together with the values of $a$ and $b$, and noting that

$$
b=\frac{v_{0 y}}{v_{0 x}}=\tan \theta_{0}
$$

after some simple algebraic manipulations we find

$$
s_{\text {ave }}=\frac{v_{0}}{2}\left[1+\frac{\cos ^{2} \theta_{0}}{\sin \theta_{0}} \ln \left(\frac{1+\sin \theta_{0}}{\cos \theta_{0}}\right)\right]
$$

Equation (16) describes the average speed of a projectile in the absence of air resistance in terms of its initial speed and the launch angle. In fact, any projectile motion is completely defined by these two parameters. An inspection of the second term on the right-hand side of this equation shows that it becomes indeterminate for $\theta_{0}=0$ and $\theta_{0}=\pi / 2$ because

$$
\lim _{\theta_{0} \rightarrow 0} \frac{\cos ^{2} \theta_{0}}{\sin \theta_{0}} \ln \left(\frac{1+\sin \theta_{0}}{\cos \theta_{0}}\right)=\frac{0}{0}
$$

and

$$
\lim _{\theta_{0} \rightarrow \pi / 2} \frac{\cos ^{2} \theta_{0}}{\sin \theta_{0}} \ln \left(\frac{1+\sin \theta_{0}}{\cos \theta_{0}}\right)=0 \times \infty
$$

However, one can use the L'Hôpital rule to evaluate these limits, which turn out to be 1 and 0 , respectively. Therefore, for $\theta_{0}=0$ and $\theta_{0}=\pi / 2$, we obtain $s_{\text {ave }}=v_{0}$ and $s_{\text {ave }}=v_{0} / 2$ from Equation (16), respectively. Remembering that in our analysis we have assumed that the projectile returns to the same level that it started, these results can easily be verified using Equations (4). A graph of the average speed as a function of launch angle is shown in Figure 2.

\subsection{Time-Average Instantaneous Speed}

From Equation (4), the instantaneous speed of a projectile at a time $t$ is given by

$$
v=\sqrt{v_{0 x}^{2}+\left(g t-v_{0 y}\right)^{2}}
$$

Using the definition of the average of a function $f(x)$ over an interval $[a, b]$ [15],

$$
\bar{f}_{a b}=\frac{1}{b-a} \int_{a}^{b} f(x) \mathrm{d} x
$$




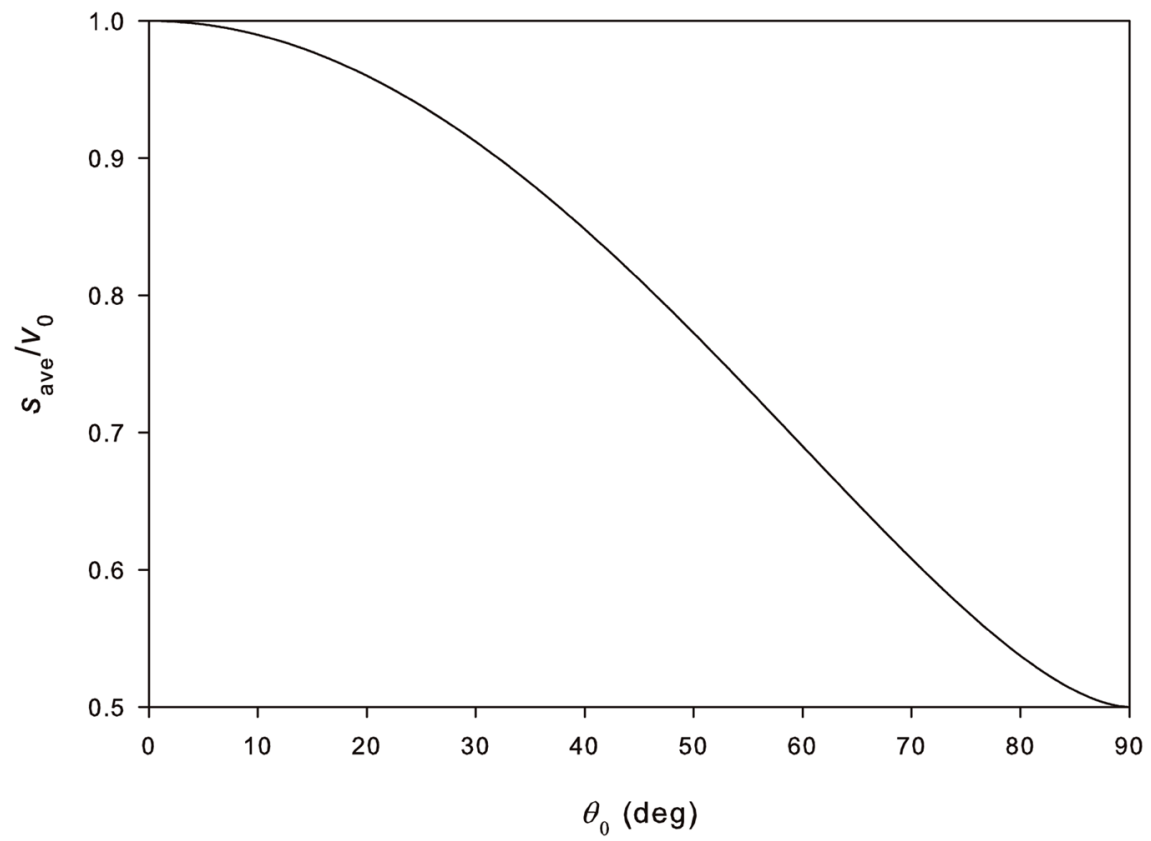

Figure 2. Average speed of a projectile as a function of launch angle according to Equation (16).

we calculate the time-average of the instantaneous speed of the projectile during its motion,

$$
\bar{v}_{t}=\frac{1}{T} \int_{0}^{T} \sqrt{v_{0 x}^{2}+\left(g t-v_{0 y}\right)^{2}} \mathrm{~d} t
$$

where the time of flight $T$ is given by Equation (13). Substituting for $T$ and changing the variable of integration to $u=g t-v_{0 y}$, we obtain

$$
\bar{v}_{t}=\frac{1}{v_{0 y}} \int_{0}^{v_{0} y} \sqrt{v_{0 x}^{2}+u^{2}} \mathrm{~d} u
$$

Evaluation of this integral is again elementary and the result is

$$
\bar{v}_{t}=\frac{1}{2}\left[\sqrt{v_{0 x}^{2}+v_{0 y}^{2}}+\frac{v_{0 x}^{2}}{v_{0 y}} \ln \left(\frac{v_{0 y}+\sqrt{v_{0 x}^{2}+v_{0 y}^{2}}}{v_{0 x}}\right)\right]
$$

Substituting $v_{0}^{2}=v_{0 x}^{2}+v_{0 y}^{2}, \quad v_{0 x}=v_{0} \cos \theta_{0}$, and $v_{0 y}=v_{0} \sin \theta_{0}$, this equation reduces to Equation (16). Therefore, the average speed and the time-average instantaneous speed of a projectile in the absence of air resistance are the same.

\subsection{Space-Average Instantaneous Speed}

According to the law of conservation of mechanical energy, the speed of a projectile in the absence of air resistance is a function of its height, $y$. Therefore, the space dependence of the speed of the projectile is given by

$$
v=\sqrt{v_{0}^{2}-2 g y}
$$

and from Equation (4) the maximum height reached by the projectile is 


$$
y_{\max }=\frac{v_{0}^{2} \sin ^{2} \theta_{0}}{2 g}
$$

Therefore, the space-average of the instantaneous speed is obtained from

$$
\bar{v}_{s}=\frac{1}{y_{\max }} \int_{0}^{y_{\max }} \sqrt{v_{0}^{2}-2 g y} \mathrm{~d} y
$$

This integral can easily be evaluated and, after substituting for $y_{\max }$ from Equation (25) and some algebraic manipulations, we obtain

$$
\bar{v}_{s}=\frac{2 v_{0}}{3 \sin ^{2} \theta_{0}}\left(1-\cos ^{3} \theta_{0}\right)
$$

which gives the space-average instantaneous speed of the projectile as a function its initial speed and launch angle. A graph of this equation together with that of Equation (16) are shown in Figure 3 for comparison. As can be seen, these two speeds are different. Therefore, the space-average of instantaneous speed of the projectile is not equal to its average speed. Again, we point out that Equation (27) becomes indeterminate $(0 / 0)$ at $\theta_{0}=0$, but

$$
\lim _{\theta_{0} \rightarrow 0} \bar{v}_{s}=v_{0}
$$

\section{Average Speed, Time-Average Instantaneous Speed, and Space-Average Instantaneous Speed in General Motion}

We now show that the average speed and the time-average instantaneous speed of a particle in any general motion are always the same but they are different from the space-average instantaneous speed.

Consider the general motion of a particle in three dimensions, described by its

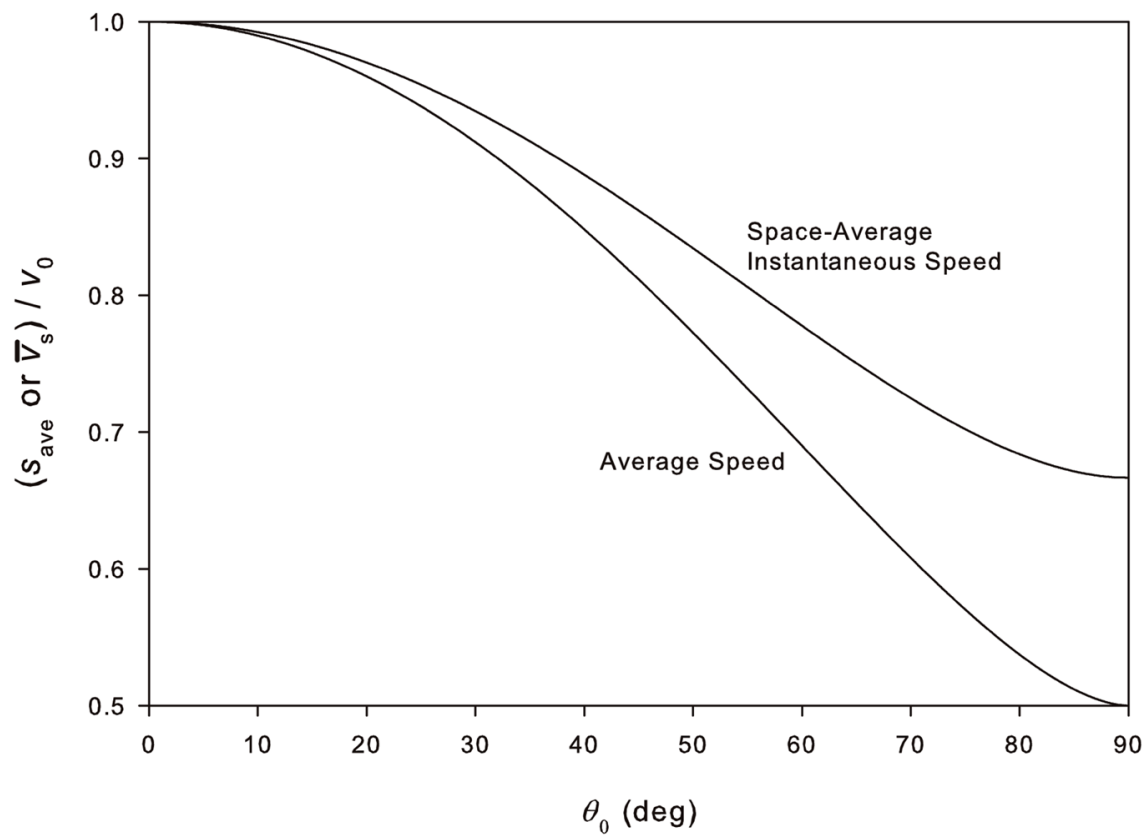

Figure 3. Average speed and space-average instantaneous speed of a projectile as a function of launch angle. 
position vector $\mathbf{r}=\mathbf{r}(t)$. The total distance that the particle travels in a time interval $t_{2}-t_{1}$ is given by

$$
L=\int_{t_{1}}^{t_{2}}|\mathrm{~d} \mathbf{r}(t)|
$$

and the average speed is

$$
S_{\text {ave }}=\frac{L}{t_{2}-t_{1}}=\frac{1}{t_{2}-t_{1}} \int_{t_{1}}^{t_{2}}|\mathbf{d} \mathbf{r}(t)|
$$

On the other hand, the instantaneous speed (or magnitude of velocity) of the particle at any time is given by

$$
v=\left|\frac{\mathrm{d} \mathbf{r}(t)}{\mathrm{d} t}\right|
$$

and the time-average of the instantaneous speed during the time interval $t_{2}-t_{1}$ is

$$
\bar{v}_{t}=\frac{1}{t_{2}-t_{1}} \int_{t_{1}}^{t_{2}} v \mathrm{~d} t=\frac{1}{t_{2}-t_{1}} \int_{t_{1}}^{t_{2}}\left|\frac{\mathrm{d} \mathbf{r}(t)}{\mathrm{d} t}\right| \mathrm{d} t
$$

But since $\mathrm{d} t$ is a positive scalar, the right-hand side of this equation reduces to that of Equation (30). Therefore,

$$
s_{\text {ave }}=\bar{v}_{t}
$$

which means that for any general motion of a particle in any dimensions the average speed is equal to the time-average of its instantaneous speeds over any time interval.

On the other hand, the space-average instantaneous speed of a particle in its general motion is given by

$$
\bar{v}_{s}=\frac{\int_{p a t h}\left|\frac{\mathrm{d} \mathbf{r}}{\mathrm{d} t}\right| \mathrm{d} L}{\int_{p a t h} \mathrm{~d} L}
$$

where $d L$ is the element of length along the path of the particle. This results does not in general reduce to Equation (30) as evidenced by the analysis of the projectile motion.

\section{Discussion and Summary}

According to Equation (16), the average speed of a projectile in the absence of air resistance varies between $v_{0}$ and $v_{0} / 2$ depending on the launch angle $\theta_{0}$. At $\theta_{0}=0$ the average speed is maximum $\left(v_{0}\right)$ and at $\theta_{0}=90^{\circ}$ it is minimum $\left(v_{0} / 2\right)$. The average speed of $3 v_{0} / 4$, midpoint between the minimum and maximum occurs at $\theta_{0}=52.8^{\circ}$.

Direct calculations of the time-average instantaneous speed of a projectile in the absence of air resistance reveal that this speed and the average speed of the projectile are the same, but they are different from the space-average instantaneous speed. These results prompted the investigation of various average 
speeds in general motion of a particle. As it turns out, average speed and time-average instantaneous speed are exactly the same regardless of the dimensionality of the motion and the nature of the forces involved during the motion. Thus, for example, the average speed and the time-average instantaneous speed of a projectile during its motion are equal even in the presence of air resistance. The space-average instantaneous speed, on the other hand, is not equal to the other two.

The equality of average speed and time-average instantaneous speed can be useful in situations where the calculation of one is more difficult than the other. For example, if the equation of the trajectory of planar motion of a particle $y=f(x)$ and a given time interval $\left[t_{1}, t_{2}\right]$ are known, one can calculate the time-average instantaneous speed by calculating the average speed. Direct calculation of time-average instantaneous speed in this case would otherwise be more involved.

This work is intended to complement the existing wealth of literature on projectile motion as well as proving that the average speed and the time-average instantaneous speed of a particle are always the same in any general motion, but different from the space-average instantaneous speed.

\section{Conflicts of Interest}

The authors declare no conflicts of interest regarding the publication of this paper.

\section{References}

[1] Halliday, D., Resnick, R. and Walker, J. (2005) Fundamentals of Physics. 7th Edition, Wiley, New York, 64-67.

[2] Serway, R.A. and Jewett Jr., J.W. (2014) Physics for Scientists and Engineers. 9th Edition, Brooks/Colem Boston, 84-91.

[3] Knight, R.D. (2008) Physics for Scientists and Engineers. 2nd Edition, Pearson/Addison-Wesley, San Francisco, 97-102.

[4] Weidner, R.T. (1985) Physics. Allyn \& Bacon, Massachusetts, 68-73.

[5] Tipler, P.A. and Mosca, G. (2004) Physics for Scientists and Engineers. 5th Edition, Volume 1, Freeman and Company, New York, 65-72.

[6] Fishbane, P.M., Gasiorowicz, S. and Thornton, S.T. (1993) Physics for Scientists and Engineers. Prentice-Hall, Englewood Cliffs, 71-76.

[7] Giancoli, D.C. (1980) Physics. Prentice-Hall, Englewood Cliffs, 51-54.

[8] Giambattista, A., McCarthy-Richardson, B. and Richardson, R.C. (2017) College Physics. McGraw-Hill, New York, 120-126.

[9] Mohazzabi, P. and Kohneh, Z.A. (2005) Projectile Motion without Trigonometric Functions. The Physics Teacher, 43, 114-115. https://doi.org/10.1119/1.1855750

[10] Barger, V.D. and Olsson, M.G. (1995) Classical Mechanics. 2nd Edition, McGraw-Hill, New York, 30-31.

[11] Fowles, G.R. and Cassiday, G.L. (1999) Analytical Mechanics. 6th Edition, Sanders, New York, 145-153. 
[12] Mohazzabi, P. (2018) When Does Air Resistance Become Significant in Projectile Motion? The Physics Teacher, 56, 168-169. https://doi.org/10.1119/1.5025298

[13] Mohazzabi, P. and Fields, J.C. (2004) High-Altitude Projectile Motion. Canadian Journal of Physics, 82, 197-204. https://doi.org/10.1139/p04-001

[14] Korn, G.A. and Korn, T.M. (1968) Mathematical Handbook for Scientists and Engineers. 2nd Enlarged and Revised Edition, McGraw-Hill, New York, 943.

[15] Stewart, J. (2016) Single Variable Calculus: Early Transcendentals. 8th Edition, Cengage, Boston, 461. 